\title{
In Vivo Quantification of Ethanol Kinetics in Rat Brain
}

\author{
Elfar Adalsteinsson 1,2, Edith V Sullivan ${ }^{3}$, Dirk Mayer ${ }^{4}$ and Adolf Pfefferbaum*,3,5 \\ 'Harvard-MIT Division of Health Sciences and Technology, MIT, Cambridge, MA, USA; ${ }^{2}$ Department of Electrical Engineering and Computer \\ Science, MIT, Cambridge, MA, USA; ${ }^{3}$ Department of Psychiatry and Behavioral Sciences, Stanford University School of Medicine, Stanford, CA, \\ USA; ${ }^{4}$ Department of Radiology, Stanford University School of Medicine, Stanford, CA, USA; ${ }^{5}$ Neuroscience Program, SRI International, Menlo \\ Park, CA, USA
}

\begin{abstract}
Proton magnetic resonance spectroscopy was used at $3 T$ to measure the uptake and clearance of brain ethanol in rats after bolus intraperitoneal (i.p.) or intragastric (i.g.) alcohol injection, and to estimate the effects of acute alcohol on brain metabolites. The observation duration was $1-1.5 \mathrm{~h}$ with temporal resolution of alcohol sampling ranging from $4 \mathrm{~s}-4 \mathrm{~min}$. The observed time course of alcohol brain concentration followed a consistent pattern characterized by a rapid absorption, an intermediate distribution, and a slower clearance that approached a linear decay. In a sample of eight healthy Wistar rats, the intercept of the linear clearance term, extrapolated back to the time of injection, correlated well with the administered dose per unit of lean body mass. Alcohol concentration estimation based on spectroscopically measured clearance was compared with blood alcohol levels from blood samples at the end of observation, and were in good agreement with the administered dose. Serial proton spectroscopy measurements provide a valid in vivo method for quantifying brain alcohol uptake and elimination kinetics in real time.

Neuropsychopharmacology (2006) 3 I, 2683-269I. doi:I 0. I038/sj.npp. I 30 I023; published online I I January 2006
\end{abstract}

Keywords: MR spectroscopy; alcohol; ethanol; glutamate; rat; imaging

\section{INTRODUCTION}

Alcohol consumption has acute effects on brain function, indicating direct action on brain tissue, neurotransmitter systems, and metabolism. Early human studies on the kinetics of alcohol metabolism assayed blood or breath, and observed that the time course of alcohol uptake and elimination varies with many factors, most notably, dose per body composition and mass, sex, type, and recency of food eaten (Erickson, 1976; Grant et al, 2000; Kalant, 1971; Li et al, 2000; O'Connor et al, 1998; Tabakoff and Hoffman, 1996; Widmark, 1981 (originally published 1932)). More recently, genetic factors have been suggested as determinants in alcohol clearance. For example, in Jewish men, a functional role has been demonstrated for the $\mathrm{ADH} 22^{*}$ allele of the alcohol dehydrogenase enzyme $(\mathrm{ADH})$, with $\mathrm{ADH} 2 * 2$ carriers having significantly higher alcoholelimination rates than $\mathrm{ADH} 2{ }^{\star} 1$ homozygotes. Accelerated elimination of alcohol may serve as a protective factor in the development of alcoholism, the prevalence of which is relatively low in Jewish men (Neumark et al, 2004). Breath

\footnotetext{
*Correspondence: Dr A Pfefferbaum, Neuroscience Program, SRI International, 333 Ravenswood Avenue, Menlo Park, CA 94025, USA, Tel: + 650859 2927, Fax: + 6508592743 ,

E-mail: dolf@synapse.sri.com

Received 15 July 2005; revised 28 November 2005; accepted 29 November 2005

Online publication: 6 December 2005 at http://www.acnp.org/citations/ Npp | 20605050458/default.pdf
}

alcohol measurements, which are readily collected in humans but challenging to accomplish in rodents, may estimate brain alcohol levels more accurately than blood levels because breath levels reflect arterial concentration of alcohol (Kalant, 1971; O'Connor et al, 1998; Ramchandani et al, 1999).

Animal studies have permitted the use of more invasive methods, such as indwelling arterial or venous catheters, and cannula placement providing direct measurement of alcohol in selective brain regions (Erickson, 1976). The resulting metabolism functions differ with the infusion route, showing an overshoot of brain concentration of alcohol with intravenous (i.v.) and intraperitoneal (i.p.) infusion and a lower peak of maximum alcohol with oral and intragastric (i.g.) administration (Hahn et al, 1997). Regardless of the infusion route, alcohol kinetics can be characterized by uptake, redistribution, and metabolism phases.

Proton magnetic resonance spectroscopy (MRS) has been used to measure brain alcohol levels non-invasively in vivo (Fein and Meyerhoff, 2000; Hanstock et al, 1990; Hetherington et al, 1999; Petroff et al, 1990; Rooney et al, 2000; Sammi et al, 2000; Spielman et al, 1993). An added benefit of MRS for the estimation of brain alcohol kinetics is the ability to monitor alcohol-induced changes in the spectroscopically visible brain metabolites during kinetics testing. The proton spectrum provides quantitative measures of vital brain metabolites: $N$-acetyl compounds, primarily $\mathrm{N}$-acetyl-aspartate (NAA), a marker of living 
mature neurons; creatine + phosphocreatine $(\mathrm{Cr})$, influenced by high-energy phosphate metabolism; cholinecontaining compounds (Cho), influenced by membrane turnover; and amino acids, primarily glutamine and glutamate (Glu). The bulk of the ethanol pool is MRSvisible via the ethanol methyl protons and can be detected by a characteristic triplet at $1.3 \mathrm{ppm}$. For rapid sampling of alcohol kinetics, conventional one-dimensional (1D) spectroscopy of the $1.3 \mathrm{ppm}$ triplet yields the highest temporal resolution. Although uncoupled spins, such as NAA, Cr, and Cho are routinely measured with 1D spectroscopy, unequivocal detection of strongly coupled and overlapping resonances, such as Glu, is more challenging.

NAA and Cho can be affected in humans with chronic alcoholism, where, following short-term (days) abstinence from alcohol, NAA and Cho may be depressed (Durazzo et al, 2004; Parks et al, 2002; Schweinsburg et al, 2001; Seitz et al, 1999) but can improve with longer-term abstinence (a month or more) (Parks et al, 2002; Schweinsburg et al, 2001). A longitudinal study of Sprague-Dawley rats chronically exposed to alcohol, however, showed an increase in the Cho/NAA ratio, peaking at about 16 weeks, followed by a decrease after 44 weeks of chronic alcohol exposure (Lee et al, 2003). Follow-up HPLC studies of tissue indicated that the alcohol-related metabolite ratio changes were possibly attributable to change in Cho rather than NAA. MRS studies of acute administration of alcohol in nonalcoholic humans have revealed that alcohol diffuses freely with water and is thus observed in concentrations proportional to the amount of water in a given brain compartment, although the variability of spectroscopically visible brain alcohol concentrations reported is between 21 and $99 \%$, depending on scanning and scanner parameters and correction approach for tissue type (Fein et al, 1995; Hetherington et al, 1999; Kaufman et al, 1996; Spielman et al, 1993).

Whether the presence of alcohol affects the MRSobserved signal from other proton metabolites has not been reported even though the quantitative study of potential effects may provide clues to biochemical mechanisms of alcohol's acute action on brain function and metabolism. Glu, an excitatory neurotransmitter present throughout the brain, is particularly relevant to the study of alcoholism, in that the glutamatergic system is differentially affected by acute and chronic alcohol exposure (Krystal et al, 2003; Melendez et al, 2005), and the modification of Glu's action may mark the development of alcohol addiction. Further, the excessive increase in extracellular Glu following withdrawal of repeated alcohol exposure induces physiological withdrawal symptoms (Becker, 1998; Dahchour and De Witte, 2003). Compared with NAA, Cr, and Cho, however, in vivo quantification of Glu is challenging at field strengths common for human imaging $(1.5$ and $3.0 \mathrm{~T})$, and requires more elaborate and timeconsuming acquisition schemes, such as CT-PRESS (Dreher and Leibfritz, 1999; Mayer and Spielman, 2005) and 2D Jresolved MRS (Hurd et al, 2004; Hurd et al, 1998; Srinivasan et al, 2005), for measurement simultaneous with alcohol kinetics. An additional benefit of 2D J-resolved MRS is the ability to measure $T_{2}$ relaxation for uncoupled spins, but measuring the kinetics of alcohol uptake with $2 \mathrm{D}$ spectroscopy levels results in a significant trade-off in temporal resolution compared to 1D spectroscopy.

Here, we performed non-invasive studies of alcohol brain kinetics and MR-visible, neurochemical effects in response to acute alcohol administration in rats. To accomplish this, we used in vivo MR spectroscopy on a human $3 \mathrm{~T}$ scanner with a custom-built RF coil. The objectives were to quantify the stages of brain alcohol following acute i.p. and i.g. infusion of $1 \mathrm{~g} / \mathrm{kg}$ ethanol using 1D spectroscopy optimized for high temporal resolution (4s). In a separate study, we used lower temporal resolution $(2.4$ and $4.7 \mathrm{~min}) 2 \mathrm{D}$ J-resolved MRS permitting longer acquisition times with higher signal-to-noise ratio (SNR), to measure concentrations of NAA, Cr, Cho, and Glu and $\mathrm{T}_{2}$-relaxation times of NAA, Cr, and Cho following IP administration of alcohol.

\section{METHODS}

\section{Subjects}

Ten adult Charles River Wistar rats ranging in weight from 504 to $671 \mathrm{~g}$, and $150-250$ days in age, were used in these studies: one rat in the high temporal resolution MRS protocol with i.p. alcohol infusion; one rat in the high temporal resolution MRS protocol with i.g. alcohol infusion; and eight rats (comprising two cohorts of four animals) in the lower temporal resolution MRS protocol with i.p. alcohol infusion.

\section{Anesthesia}

As previously described (Adalsteinsson et al, 2004; Pfefferbaum et al, 2004), the animals were held in an MR-invisible structure, which provided support for the RF coil and a nose cone for the delivery of isoflurane anesthesia $(2-3 \%)$ and oxygen $(\sim 21 / \mathrm{min})$. Rectal temperature and oxygen saturation from a hind limb were monitored throughout the experiment. Heating was provided by pre-warmed bags of saline solution placed under the animal tray as well as from a temperature-controlled water-heating blanket suspended over the animal and coil (Figure 1).

\section{Catheter Placement}

After sedation, a flexible catheter was inserted into the i.p. cavity or into the stomach via the esophagus for i.g. injection. For the i.p. protocol, before MRS acquisition with the standard gradients, $\mathrm{T}_{1}$-weighted (3-plane localizer, echo time $(\mathrm{TE}) /$ repetition time $(\mathrm{TR})=1.7 / 32.1 \mathrm{~ms}, \mathrm{FOV}=16 \mathrm{~cm}$, $5 \mathrm{~mm}$ thick, three slices/plane) multislice whole-body images were acquired with the standard human body coil before and after injection of $1 \mathrm{cc}$ of $1 \%$ Gadolinium-DTPA (Gd) in saline. Substantial signal enhancement around the bowel following the Gd injection indicated proper catheter placement (Figure 2). For the i.g. protocol, i.g. catheter placement was verified by aspiration of gastric acid.

\section{MRI and MRS}

Data were acquired on a clinical $3 \mathrm{~T}$ GE Signa human MRI scanner with standard gradients that deliver maximum amplitude of $40 \mathrm{mT} / \mathrm{m}$ and maximum slew rate of $150 \mathrm{~T} / \mathrm{m} / \mathrm{s}$. 
A custom-made rat brain quadrature head coil was used for excitation and reception of proton signals. An animal gradient insert (Chronik et al, 2000a; Chronik et al, 2000b; Foster-Gareau et al, 2003; Heyn et al, 2005) with a $20 \mathrm{~cm}$ bore, a maximum amplitude of $500 \mathrm{mT} / \mathrm{m}$, and a slew rate of $1875 \mathrm{~T} / \mathrm{m} / \mathrm{s}$ was used in the comparison study of i.g. and i.p. infusion.

A 3-plane localizer scan $(\mathrm{TE} / \mathrm{TR}=2.1 / 54 \mathrm{~ms}, \mathrm{FOV}=$ $80 \mathrm{~mm}, 256 \times 128,5 \mathrm{~mm}$ thick, 10 slices/plane) was used for the prescription of coronal fast spin-echo (FSE) images $(\mathrm{TE} 1 / \mathrm{TE} 2 / \mathrm{TR}=18 / 90 / 2200 \mathrm{~ms}, \quad \mathrm{FOV}=40 \mathrm{~mm}, 256 \times 256$, $2.5 \mathrm{~mm}$ thick, 12 slices). An approximately $0.25 \mathrm{cc}$ brain voxel $\left(10 \times 5 \times 5 \mathrm{~mm}^{3}\right)$ was prescribed from the FSE section that allowed the largest in-plane voxel placement (Figure 3),

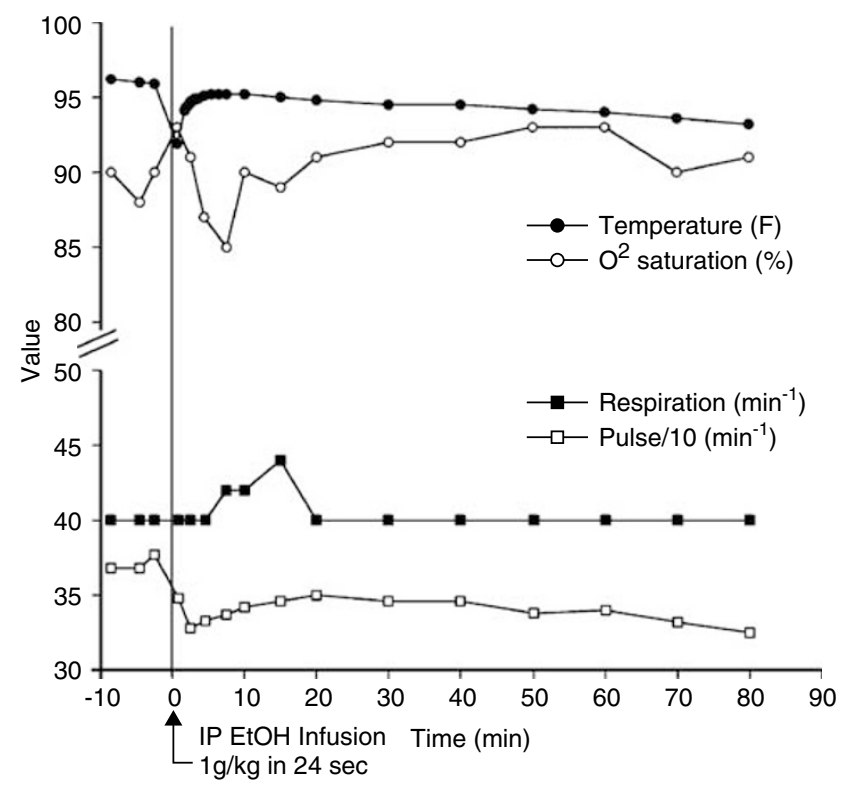

Figure I An example from one animal of the evolution of monitored temperature $(\mathrm{F}), \mathrm{O}_{2}$ saturation (\%), respiration rate $\left(\mathrm{min}^{-1}\right)$, and heart rate $\left(\mathrm{min}^{-1}\right)$ after subtraction by 300 to share the numerical values on vertical scale with the respiration. The drop in core temperature (gray points and curve) of the rat was caused by the room temperature injection.

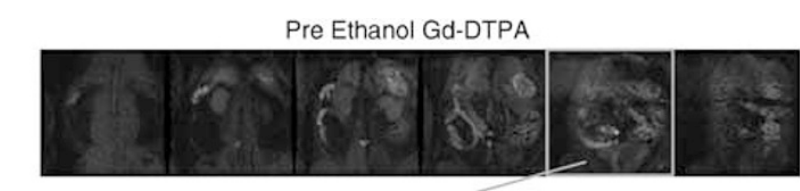

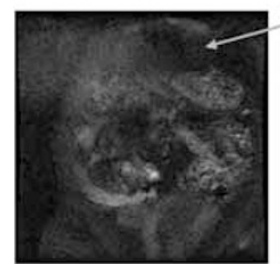

Pre-injection

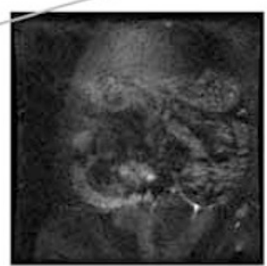

Post 1cc 1\%Magnevist

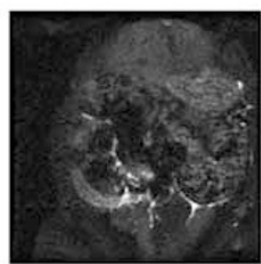

Post 2cc 1\%Magnevist
Figure 2 An example of verification of i.p. catheter placement by injection of I and $2 \mathrm{cc}$ of $1 \%$ Gd-DTPA (Magnevist). The enhancement of signal around the bowel indicates that the catheter is indeed residing in the i.p. cavity. and a series of single-voxel spectra were acquired before and after bolus injection of $1 \mathrm{~g}$ ethanol $/ \mathrm{kg}$ of total body weight at room temperature in approximately $5 \mathrm{cc}$ volume. Total duration of MRS observation was $1-1.5 \mathrm{~h}$. For the high-resolution i.g. and i.p. studies, conventional 1D PRESS spectra at $\mathrm{TE}=144 \mathrm{~ms}$ were acquired with two averages and a $2 \mathrm{~s}$ TR to yield a $4 \mathrm{~s}$ temporal resolution of the alcohol triplet at $1.3 \mathrm{ppm}$. For the lower time resolution 2D J-resolved MRS study, we used $4.7 \mathrm{~min}$ acquisitions for three animals (Dreher and Leibfritz, 1995; Hurd et al, 1998) $(\mathrm{TE} 1 / \mathrm{TR}=35 / 2000 \mathrm{~ms}, \mathrm{TE}=10 \mathrm{~ms}, 16 \mathrm{TE}$ steps, eight averages per TE step) (Figure 4) and $2.4 \mathrm{~min}$ for five animals (2D J-resolved MRS, same parameters, except with four averages per TE step). Water suppression was performed with CHESS (Haase et al, 1985), with flip angles determined by the default GE PRESS sequence. These water suppression settings were found to pass through adequate amount of residual water (between $10: 1$ and $100: 1$ relative to NAA) to provide phasing of the metabolite spectra for each TE. Each readout was sampled with 2048 points at a $5 \mathrm{kHz}$ bandwidth. Scan sessions were approximately $2 \mathrm{~h}$ per animal and spanned pre- and post-infusion periods.

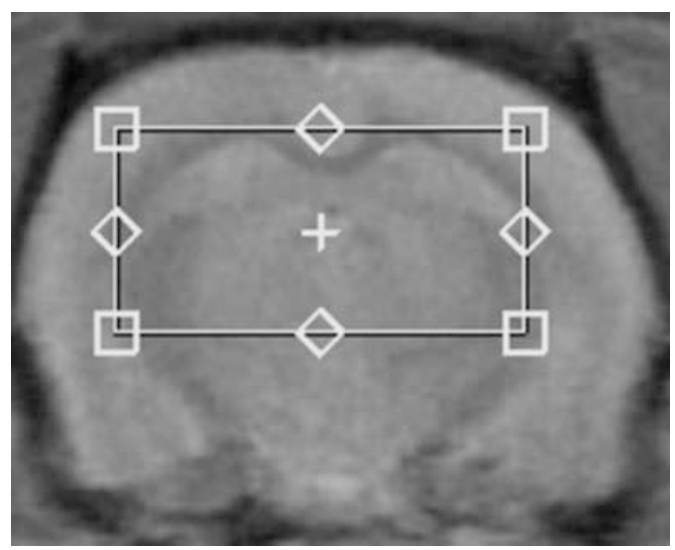

Figure 3 Location of PRESS voxel on an FSE scout image. Voxel dimensions were approximately $10 \mathrm{~mm}(\mathrm{~L}-\mathrm{R}) \times 5 \times 5 \mathrm{~mm}$.

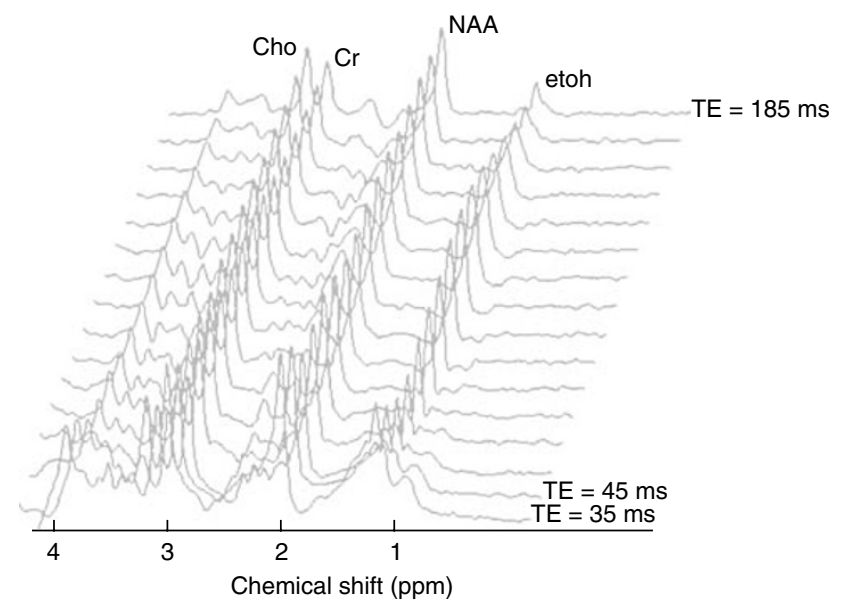

Figure 4 The 16 spectra acquired as part of one 2D, J-resolved experiment for an animal. Initial $T E=35 \mathrm{~ms}, 10 \mathrm{~ms}$ interval, final $\mathrm{TE}=185 \mathrm{~ms}$, eight signal averages per spectrum. 


\section{Spectral Processing}

The data with partial water suppression were phase corrected based on residual water as described by Webb et al (1994). Briefly, a zero and first-order phase terms were extracted and the signal phase unwrapped, followed by a smoothing spline fit to any residual nonlinear phase component, that was then removed. The time-domain data were then apodized with a $6 \mathrm{~Hz}$ Gaussian filter, zero filled to 4096 points and Fourier transformed. Next, residual water and water sidebands were removed from the partially suppressed metabolite data through pure water subtraction (Adalsteinsson et al, 2004; Hurd, 2000). A single linear term spanning the entire spectral bandwidth was then removed based on a fit to data in spectral bins near the highest and lowest frequencies $( \pm 2.5 \mathrm{kHz})$. A final frequency correction step was performed to compensate for small temperature variations during the scans by referencing each frame to NAA at $2.02 \mathrm{ppm}$.

For each 2D acquisition, the TE-averaged spectrum, that is, the average of the spectra from all TE values, was used for the quantification of metabolites and for ethanol at $1.3 \mathrm{ppm}$. This corresponds to only using the center line from a 2D J-resolved spectrum for the analysis. Peak integration by fitting to a Gaussian line shape was used to quantify the spectral peaks in the TE-averaged spectra. The spectra for each rat were normalized by the amount of unsuppressed water in the voxel, estimated by fitting a twoterm exponential decay to the unsuppressed water signal for the 16 echo times in a $2 \mathrm{D}$ J-resolved acquisition sampled at baseline before alcohol injection. To convert ethanol peak areas to standard units, the average NAA across the eight rats used for the lower temporal resolution i.p. study was assumed to be $9 \mathrm{mM}$. For the single-echo PRESS acquisitions of the highest temporal resolution i.p. and i.g. data, the ethanol triplet at $1.3 \mathrm{ppm}$ was integrated and NAA in each of the animals was assumed to be $9 \mathrm{mM}$. No corrections were made for potential relaxation time differences between the $\mathrm{NAA}$ and $\mathrm{EtOH}$ peaks.

A parametric curve consisting of two exponentials and a single linear term, $\alpha+\beta t+a_{\mathrm{d}} e^{\left(-k_{\mathrm{d}} t\right)}-a_{\mathrm{a}} e^{\left(-k_{\mathrm{a}} t\right)}$, was fitted to the temporal sequence of the $1.3 \mathrm{ppm}$ ethanol estimates, where $\alpha$ and $\beta$ are the intercept and slope of the linear component of the fit; $k_{\mathrm{a}}$ and $k_{\mathrm{d}}$ are the time constants associated with the rapid initial absorption and the subsequent slower distribution; and $a_{\mathrm{a}}$ and $a_{\mathrm{d}}$ are the corresponding amplitude factors. The nonlinear, leastsquares fit was performed in Matlab (Mathworks, Cambridge, MA) with initial values that provided a good fit to a typical curve from the data set. Alcohol-related parameters derived from the model included time-to-peak (time from the start of the bolus injection to the peak of the fitted curve); intercept ( $\alpha$, determined from the Widmark constant, the linear component of the fit projected back to time $=0$, that is, start of bolus injection); the alcohol overshoot (the area below the fitted curve, but above the linear component); and elimination rate (slope of the linear component of the fit).

The effect of alcohol infusion on peak areas of the singlet resonances of $\mathrm{NAA}$ at $2.0 \mathrm{ppm}, \mathrm{Cr}$ at $3.0 \mathrm{ppm}$, Cho at $3.2 \mathrm{ppm}$, the Glu C4 resonance at $2.4 \mathrm{ppm}$, and $\mathrm{T}_{2}$ of NAA, $\mathrm{Cr}$, and Cho, was examined by comparing the metabolite values at baseline and the common, final post-infusion time available for all eight rats. For each animal, the baseline was chosen as the data from the $4.7 \mathrm{~min}$ data collection just before infusion; the post-infusion value was from $4.7 \mathrm{~min}$ of acquisition time at $\sim 37 \mathrm{~min}$ after infusion. The spectra were averaged over echo times $55-185 \mathrm{~ms}$, and individual metabolites estimated with a Gaussian fit ( $0.14 \mathrm{ppm}$ width).

An independent estimate of the alcohol concentration was determined for four animals from retro-orbital blood drawn within $5 \mathrm{~min}$ of the end of the MRS session.

\section{RESULTS}

\section{Alcohol Kinetics}

High temporal resolution of alcohol kinetics for i.p. and i.g. infusion. In two animals, we compared the time courses of the spectroscopically visible brain alcohol signal following i.p. $v s$ i.g. bolus of $1 \mathrm{~g} / \mathrm{kg}$ ethanol. Figure 5a shows the integrated area of the methyl triplet (dots) at $1.3 \mathrm{ppm}$, as a function of time following i.p. bolus injection (i.p.: intercept $=41 \mathrm{mg} \%$, slope $=-0.21 \mathrm{mg} \% / \mathrm{min}$, $k_{\mathrm{a}}=0.33 \mathrm{~min}^{-1}, \quad k_{\mathrm{d}}=0.13 \mathrm{~min}^{-1}, \quad a_{\mathrm{a}}=104 \mathrm{mg} \%, \quad a_{\mathrm{d}}=$ $63 \mathrm{mg} \%$, overshoot area $=226 \mathrm{mg} \%^{*} \min , t_{\max }=6.9 \mathrm{~min}$, $\max =56 \mathrm{mg} \%)$. The data were characterized by a threephase process of a rapid uptake, an exponential initial decay, and a longer-term linear decay in brain alcohol signal. Figure $5 \mathrm{~b}$ shows brain alcohol data following an i.g. ethanol bolus, and were also described in terms of a threephase time course (i.g.: intercept $=17 \mathrm{mg} \%$, slope $=$ $-0.042 \mathrm{mg} \% / \mathrm{min}, \quad k_{\mathrm{a}}=0.24 \mathrm{~min}^{-1}, \quad k_{\mathrm{d}}=0.12 \mathrm{~min}^{-1}, \quad a_{\mathrm{a}}=$ $33 \mathrm{mg} \%, \quad a_{\mathrm{d}}=16 \mathrm{mg} \%$, overshoot area $=31 \mathrm{mg} \%{ }^{\star} \min$, $\left.t_{\max }=11.2 \mathrm{~min}, \max =19 \mathrm{mg} \%\right)$. The brain EtOH uptake following i.g. administration, however, was slower than that for i.p. administration (time-to-peak $=11.2 \mathrm{~min}$ for i.g. $v s$ $6.9 \mathrm{~min}$ for i.p.), and the data displayed a less exaggerated peak signal relative to the linear decay phase (overshoot area 31 compared to $225 \mathrm{mg} \%{ }^{\star} \mathrm{min}$ ).

Lower temporal resolution of alcohol kinetics and spectral peak analysis. Reducing temporal resolution of alcohol kinetics from seconds to minutes enables an improved quantification of the effects of acute alcohol administration on other MRS-visible compounds. For this study, eight rats were scanned with a 2D J-resolved acquisition with 16 echo times; for three rats, the temporal resolution for alcohol kinetics was $4.7 \mathrm{~min}$, and for five rats, it was $2.4 \mathrm{~min}$. An example of each sampling scheme is presented in Figure 6 and demonstrates similar three-phase evolution of brain alcohol as observed in the highest $(4 \mathrm{~s})$ temporal resolution, but with the expected trade-off in variance $v s$ measurement time for the individual samples.

The alcohol kinetics metrics for each of these eight rats and their mean \pm standard deviation (SD) are given in Table 1. It was expected that the estimated brain dose, as represented by the intercept of the linear component at time $=0$, would correlate with the amount of alcohol delivered. This expectation was confirmed by a significant positive correlation (Pearson $r=0.81, p=0.015$; Spearman's Rho $=0.69, p=0.068$, two-tailed) (Figure 7).

Retro-orbital blood was drawn at the end of the MRS session and alcohol concentration determined for four 


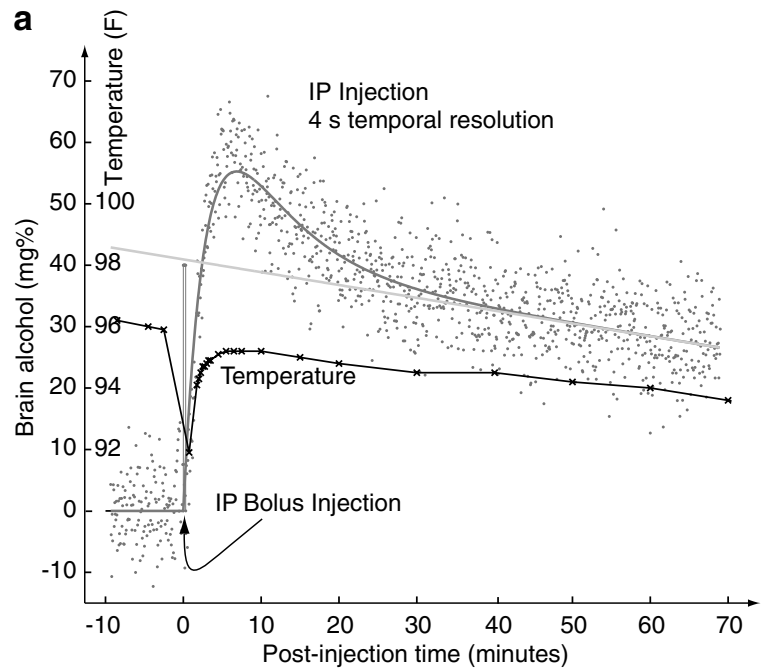

b

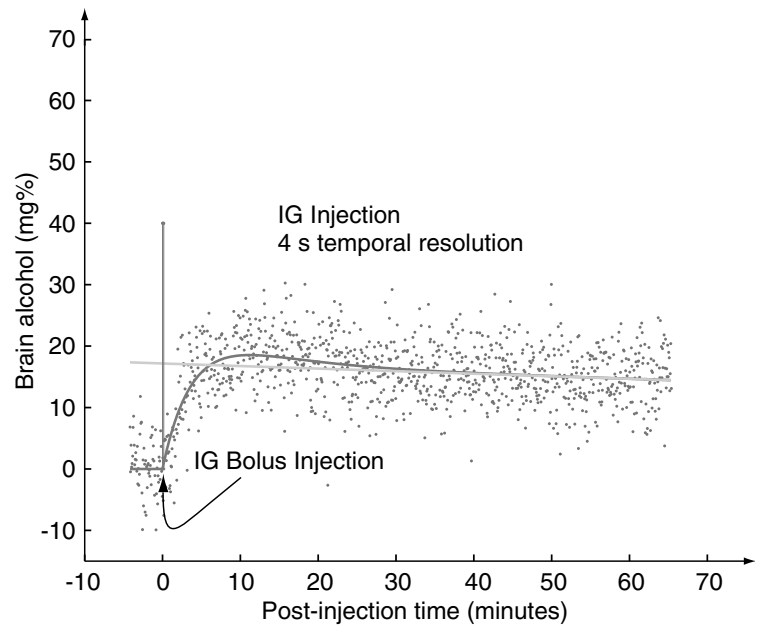

Figure 5 Examples of a $4 \mathrm{~s}$ temporal resolution sampling of brain alcohol following an i.p. (a) and i.g. (b) alcohol infusion. The data demonstrate marked flattening of the rapid uptake component characteristic of i.g. relative to i.p. infusion. Also note the sharp drop in core temperature (black x-points and curve) of the rat caused by the room temperature injection. The temperature scale (in Fahrenheit) is provided to the right on the vertical axis.

animals. Using the slope and intercept from the parametric fit to the uptake curve, combined with blood-draw time, lean body mass, and alcohol concentration at blood draw time, the estimated theoretical concentration at time zero was $139 \mathrm{mg} / 100 \mathrm{ml}(\mathrm{SD}=23.2)$. For comparison, the MRS estimated dose/lean body mass for these four rats was $132 / 100 \mathrm{gm}(\mathrm{SD}=9.8)$. Lean body mass was assumed to be approximately the same for all animals at $80 \%$ of weight for the lightest rat.

Metabolite quantification before and after alcohol infusion. $\mathrm{T}_{2}$ for $\mathrm{NAA}, \mathrm{Cr}$, and Cho were estimated by single exponential fits to the integrated spectral peaks at the 16 echo times of the 2D J-resolved acquisition (Figure 4). To determine whether the acute i.p. alcohol administration affected the $\mathrm{T}_{2}$ of the metabolites, we compared relaxation times for these three metabolite peaks sampled for $\sim 4 \mathrm{~min}$ immediately before and $37 \mathrm{~min}$ after i.p. alcohol infusion for all eight rats. This analysis failed to yield any statistically significant differences (all $p$-values $>0.17$ ).

Similarly, we tested whether the acute i.p. alcohol administration affected the integrated areas of spectral peaks for NAA, Cr, Cho, and Glu, compared at the same pre- and post-time intervals as done for the $\mathrm{T}_{2}$ comparisons. Exploratory paired t-tests $(\alpha=0.05$, two-tailed $)$ yielded decrease in Cho $(\mathrm{t}(7)=2.37, p<0.05)$ and increase in Glu $(\mathrm{t}(7)=2.65, p<.035)$ with no substantial changes in NAA $(\mathrm{t}(7)=0.931, \mathrm{NS})$ or $\mathrm{Cr}(\mathrm{t}(7)=1.36$, NS) (Figure 8). None of these differences would reach statistical significance with Bonferroni correction for four, two-tailed comparisons, requiring $p=0.0125$.

In a subgroup of four animals, 2D J-resolved spectroscopy of unsuppressed water (ie tissue water) was acquired preand post-alcohol infusion. A comparison of $\mathrm{T}_{2}$ for tissue water (the ventricular and sulcal CSF contribution was minimized by using a bi-exponential fit) showed that postinfusion $\mathrm{T}_{2}$ was slightly elevated in three animals $(1,3,4 \%)$ and decreased $(-4 \%)$ in one animal. Given the variability of these results, we cannot make any conclusions about the effect of relaxation of tissue water.

\section{DISCUSSION}

The data demonstrate the feasibility of quantifying the stages of in vivo brain alcohol kinetics in small animals while monitoring the acute effects of alcohol on brain metabolites. The high correlation of the linear intercept with alcohol dose per lean body mass along with the MRS estimates of initial dose based on blood samples indicate that this MRS procedure provides a valid in vivo method for quantifying alcohol uptake and elimination kinetics and relative brain ethanol concentration. The MRS-derived alcohol kinetics functions based on high temporal sampling rates in $4 \mathrm{~s}$ intervals are consistent with findings based on indwelling brain microdialysis, showing the characteristically sharp overshoot peak of measurable alcohol in the brain following i.p. alcohol infusion; by contrast, i.g. infusion produces a modest overshoot. The alcohol elimination arm of the kinetics function for both infusion routes can be described by the Widmark function, which is a linear component with negative, shallow slope (Kalant, 1971).

The in vivo kinetics of brain alcohol have been studied in human brain with $1 \mathrm{H}$ spectroscopic imaging at a $10 \mathrm{~min}$ (Hetherington et al, 1999) and a $13 \mathrm{~min}$ (Fein and Meyerhoff, 2000) temporal resolution, and with single-voxel spectroscopy at $4 \mathrm{~min}$ resolution (Fein and Meyerhoff, 2000). With sufficient SNR, higher temporalresolution kinetic studies are feasible, ultimately limited only by the repetition time of the acquisition, which is typically of the order of $2 \mathrm{~s}$.

The temporal characteristics observed for the i.g. infusion are in good agreement with human studies. Grant et al (2000) administered a standard oral dose of $0.75 \mathrm{~g} / \mathrm{kg}$ 


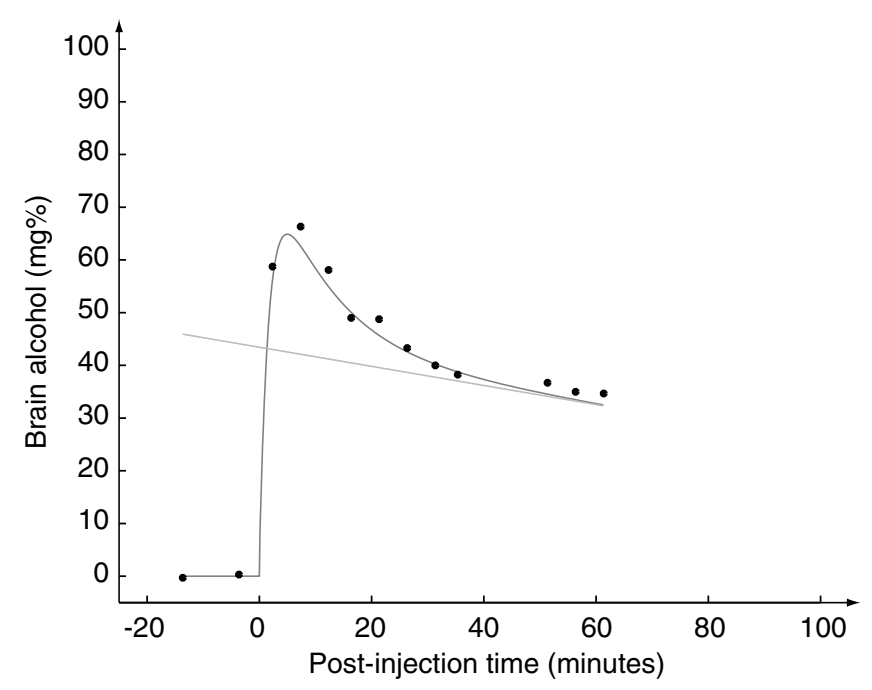

alcohol to humans, and obtained $32 \mathrm{mg} \%$ for peak bloodalcohol, about half the concentration of the initial dose. They also observed slow and continuous absorption, almost matching elimination. The expected brain concentration of alcohol after i.p. injection is governed primarily by absorption in the first 5-10 min, followed by a combination of absorption, metabolism and elimination, and after 30 min, primarily elimination.

Prior to each alcohol infusion experiment for each rat, we infused Gd-DTPA via the catheter used for ethanol injection and performed whole-body imaging to verify i.p. placement because it has been estimated that upwards of $20 \%$ of i.p. needles are inaccurately placed (Nebendahl, 2000). Any effect of Gd-DTPA on the data sampled from the selected brain volume is expected to be negligible because Gd-DTPA does not cross the blood-brain barrier under normal

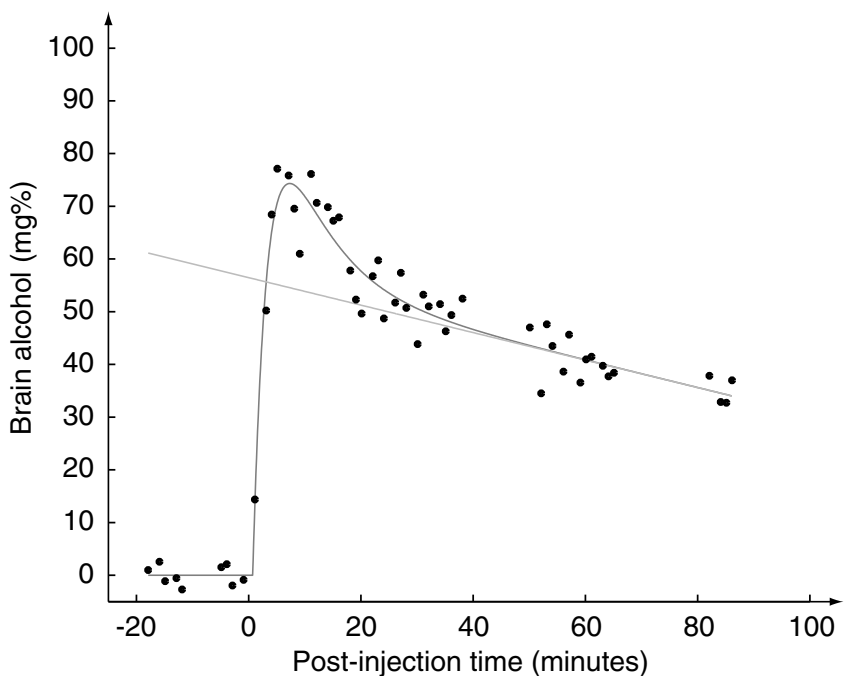

Figure $\mathbf{6}$ The MRS-visible brain alcohol signal, recorded during approximately an hour's observation for representative data with a 4.7 and $2.4 \mathrm{~min}$ resolution following an i.p. injection of $\mathrm{I} / \mathrm{kg}$ ethanol. The measured data (dots) are fit (solid curve) with a three-term expression including rapid uptake, intermediate distribution, and a much slower linear clearance (straight, sloped line).

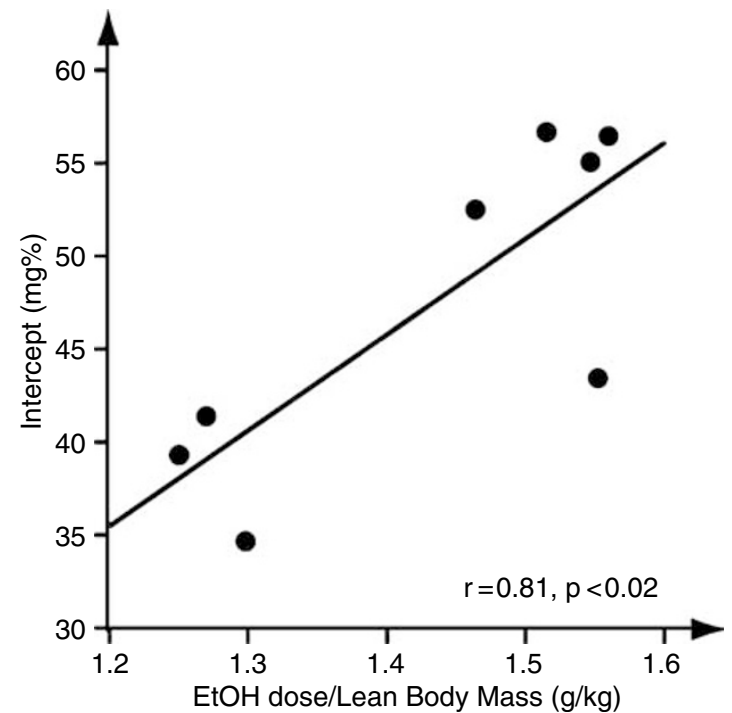

Figure 7 Correlation between the intercept, that is, the MRS estimate of the initial alcohol dose, and administered alcohol dose per unit of lean body weight. The intercept derived from the linear decay term of the kinetic fit yields an MRS estimate of the delivered alcohol dose. The intercept value correlated significantly with the administered dose per unit lean body mass.

Table I Alcohol Kinetic Parameters for Each Rat in the Lower Resolution MRS Study

\begin{tabular}{|c|c|c|c|c|c|c|c|c|c|c|}
\hline Rat & $\begin{array}{c}\text { Intercept } \\
\text { mg\% }\end{array}$ & $\begin{array}{l}\text { Slope } \\
\text { mg\%/min }\end{array}$ & $\begin{array}{c}k_{a} \\
\min ^{-1}\end{array}$ & $\begin{array}{c}k_{d} \\
\min ^{-1}\end{array}$ & $\begin{array}{c}a_{a} \\
m g \%\end{array}$ & $\begin{array}{c}a_{d} \\
m g \%\end{array}$ & $\begin{array}{c}\text { Overshoot area } \\
\text { mg\%*min }\end{array}$ & $\begin{array}{l}t_{\max } \\
\min \end{array}$ & $\begin{array}{l}\text { Max } \\
\text { mg\% }\end{array}$ & $\begin{array}{c}\text { Weight } \\
\text { g }\end{array}$ \\
\hline I & 56 & -0.13 & 0.41 & 0.07 & 170 & 78 & 753 & 7.3 & 93 & 614 \\
\hline 2 & 57 & -0.19 & 0.42 & 0.08 & 112 & 54 & 451 & 6.9 & 80 & 601 \\
\hline 3 & 43 & -0.18 & 0.59 & 0.09 & 82 & 42 & 346 & 5.0 & 65 & 616 \\
\hline 4 & 56 & -0.26 & 0.38 & 0.12 & 161 & 72 & 282 & 7.3 & 74 & 619 \\
\hline 5 & 53 & -0.27 & 0.34 & 0.17 & 99 & 41 & 47 & 8.4 & 54 & 581 \\
\hline 6 & 39 & -0.23 & 0.52 & 0.17 & 116 & 53 & 144 & 5.3 & 52 & 496 \\
\hline 7 & 35 & -0.23 & 0.41 & 0.18 & 70 & 27 & 40 & 7.0 & 37 & 515 \\
\hline 8 & 41 & -0.31 & 0.59 & 0.13 & 94 & 41 & 197 & 4.9 & 56 & 504 \\
\hline Mean & 48 & -0.22 & 0.46 & 0.13 & 113 & 51 & 282 & 6.5 & 64 & 568.3 \\
\hline SD & 9 & 0.06 & 0.10 & 0.04 & 36 & 17 & 237 & 1.3 & 18 & 53.9 \\
\hline
\end{tabular}




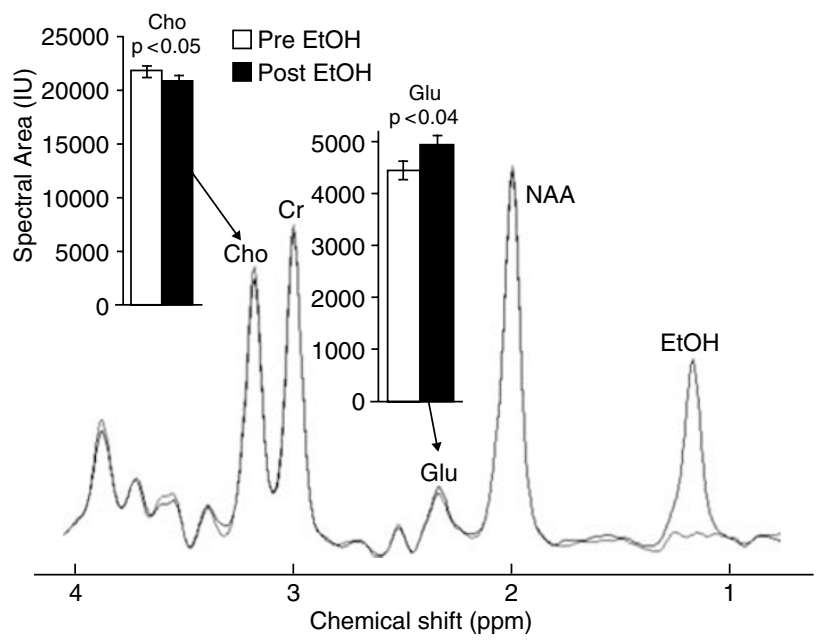

Figure 8 Mean of one-dimensional TE-averaged spectra for eight animals at baseline (gray) and approximately $37 \mathrm{~min}$ after i.p. alcohol infusion (black). Assuming a concentration of $9 \mathrm{mM}$ for NAA and ignoring relaxation differences, the mean values for Cho pre and post EtOH injection were estimated at 2.13 and $2.04 \mathrm{mM}$; for Glu they were 3.92 and $4.36 \mathrm{mM}$ under the further simplified approximation of weak coupling.

conditions. Gd-DTPA remains in the vascular pool, which makes up only a small fraction of the brain (5\% in humans), and does not cross into any of the brain constituents, whereas alcohol diffuses freely into the brain parenchyma. Consequently, we would not expect a significant contribution of the Gd-DTPA on unsuppressed water, brain metabolites, or spectroscopically visible alcohol signal from the brain volume sampled in this study.

The lower temporal resolution acquisition study, using a 2.4 or 4.7 min sampling rate with i.p. infusion, was adequate to observe the characteristic overshoot identified in the high-resolution study and permitted quantification of other metabolites. Although neither NAA nor $\mathrm{Cr}$ showed peak differences pre- $v s$ post-alcohol infusion, Cho and Glu showed modest changes, albeit not statistically significant. Conclusions about the meaning of these metabolite changes must be tempered by the fact that this was a non-blind, non-placebo, controlled observation without a priori hypotheses for Glu or Cho. MR spectroscopic estimation of brain metabolite concentrations can also be affected by changes in relaxation times, but in the current study we found only inconsistent increases in tissue water $\mathrm{T}_{2}$ (1-4\% in three of four animals), and no significant change in NAA, Cr, or Cho $\mathrm{T}_{2}$.

Among the limitations of the current study was the use of relative as opposed to absolute quantification for alcohol and metabolite concentrations. Although the use of an internal signal with an assumed concentration (in this case NAA at $9 \mathrm{mM}$ ) provides a first-order approximation to calibration in standard units, an external reference hardware-based scheme as introduced by Akoka et al (1999) could provide absolute quantitation. We also note that the duration of observation period following alcohol injection has an impact on the quantification of the parameters in the kinetic model, particularly with respect to the linear clearance term. In the group of eight rats used for the low temporal-resolution i.p. study, seven animals had data collected for $54 \mathrm{~min}$ or longer post injection, whereas one rat was observed for only $46 \mathrm{~min}$. As the accuracy of the estimate of the slope for the linear clearance term is most affected by a short observation interval, we ran a secondary analysis of the correlation between the intercept and dose per unit of lean body mass by eliminating the data point of the 46-min observation. The correlation obtained without this data point was $r=0.77, p=0.045$, which was insubstantially different from the reported correlation obtained in the full sample of eight rats $(r=0.81, p<0.02)$. Finally, the animals came in two different batches of four rats from Charles River, Inc. The first batch was older and heavier at the time of scanning and, therefore, received a larger dose / lean body weight, perhaps contributing to a greater brain alcohol concentration overshoot.

If not a chance occurrence, the unpredicted rise in the Glu peak may have implications for glutamate's role in the development of alcohol dependence. Microdialysis studies report that extracellular levels of glutamate increase with i.p. administration of low to moderate doses of ethanol (0.5-1.0 g/kg), and decrease with higher doses (Moghaddam and Bolinao, 1994a, b; Selim and Bradberry, 1996; Yan et al, 1998). Our observation of an increase in Glu resonance about $40 \mathrm{~min}$ after i.p. administration of $1.0 \mathrm{~g} / \mathrm{kg}$ is consistent with the reports using moderate doses of alcohol. Extracellular glutamate, measured by microdialysis $12-24 \mathrm{~h}$ after the last repeated alcohol administration over several days (Dahchour and De Witte, 2003; De Witte, 2004; Melendez et al, 2005; Rossetti and Carboni, 1995), is increased and thought to be a major factor in producing withdrawal symptoms (Becker, 2000). The withdrawal time increase in extracellular glutamate is usually not seen in alcohol-naive animals and only produced in animals previously repeatedly exposed to alcohol. Extracellular glutamate and its stimulation of NMDA receptors are elevated with alcohol withdrawal in certain brain regions (Roberto et al, 2004) and interactions with the glutamatergic system are of major interest in the etiology and treatment of alcoholism (Lovinger, 2002).

A number of known factors modulate the physiological effects of acute alcohol in animals and in humans, including age, sex, strain, genetics, and prior alcohol exposure (Kalant, 2000). In human populations, for example, the rate of alcohol elimination is associated with the ADH2 genotype in Jewish men, suggesting genetic as well as environmental factors contributing alcohol consumption patterns among various ethnic groups (Neumark et al, 2004). The in vivo MR spectroscopy alcohol kinetic studies applied herein in a rodent can similarly be used in human studies with oral or i.v. alcohol delivery (O'Connor et al, 1998).

In conclusion, we have developed non-invasive, real-time approaches to track the course of alcohol metabolism in the brain that can be used to characterize differences among animals selectively bred for alcohol consumption behavior (Li et al, 1979), permit safe longitudinal tracking of acute alcohol kinetics during the development and maintenance of the alcohol dependence syndrome (Koob, 2000), and can be extended to humans. With an established model of its brain kinetics, alcohol could also serve as an internal in vivo quantitative reference for spectroscopically visible metabolites. Alcohol is inexpensive, readily available, non-toxic in a wide dose range, transient with half-life on the order of 
hours, and its concentrations can be externally validated by breath and blood samples.

\section{ACKNOWLEDGEMENTS}

We acknowledge Drs William McBride and Richard Bell of the University of Indiana, who kindly supplied the animals; Marcus Alley, $\mathrm{PhD}$ and David Clayton, $\mathrm{PhD}$ for construction and calibration of the custom head coil; Diane Howard, RVT and Wendy Baumgardner, RVT, LATg, Veterinary Research Technologists, who administered anesthesia and caringly monitored the animals during scanning; Ralph Hurd, $\mathrm{PhD}$ and Napapon Sailasuta, $\mathrm{PhD}$ for scanner software support; Alexander Jack, BA, Daniel J Pfefferbaum, BA and Andrea Spadoni, BA for assistance in scan acquisition; Shay Cook, Luke Garcia, and Juan Orduna, animal technicians, who provided thoughtful care for the animals. This study was supported by grants from the National Institute on Alcohol Abuse and Alcoholism (INIA-AA13521; AA05965).

\section{REFERENCES}

Adalsteinsson E, Hurd RE, Mayer D, Sailasuta N, Sullivan EV, Pfefferbaum A (2004). In vivo 2D J-resolved magnetic resonance spectroscopy of rat brain with a $3 \mathrm{~T}$ clinical human scanner. Neuroimage 22: 381-386.

Akoka S, Barantin L, Trierweiler M (1999). Concentration measurement by proton NMR using the ERETIC method. Ann Chem 71: 2554-2557.

Becker HC (1998). Kindling in alcohol withdrawal. Alcohol Health \& Research World 22: 25-33.

Becker HC (2000). Animal models of alcohol withdrawal. Alcohol Research Health 24: 105-113.

Chronik B, Alejski A, Rutt BK (2000a). Design and fabrication of a three-axis multilayer gradient coil for magnetic resonance microscopy of mice. Magma 10: 131-146.

Chronik BA, Alejski A, Rutt BK (2000b). Design and fabrication of a three-axis edge ROU head and neck gradient coil. Magn Reson Med 44: 955-963.

Dahchour A, De Witte P (2003). Excitatory and inhibitory amino acid changes during repeated episodes of ethanol withdrawal: an in vivo microdialysis study. Eur J Pharmacol 459: 171-178.

De Witte P (2004). Imbalance between neuroexcitatory and neuroinhibitory amino acids causes craving for ethanol. Addictive Behavior 29: 1325-1339.

Dreher W, Leibfritz D (1995). On the use of two-dimensional-J NMR measurements for in vivo proton MRS: measurement of homonuclear decoupled spectra without the need for short echo times. Magn Reson Med 34: 331-337.

Dreher W, Leibfritz D (1999). Detection of homonuclear decoupled in vivo proton NMR spectra using constant time chemical shift encoding: CT-PRESS. Magn Reson Imaging 17: 141-145.

Durazzo TC, Gazdzinski S, Banys P, Meyerhoff DJ (2004). Cigarette smoking exacerbates chronic alcohol-induced brain damage: a preliminary metabolite imaging study. Alcoholism-Clin Exp Res 28: $1849-1860$.

Erickson CK (1976). Regional distribution of ethanol in the rat brain. Life Sci 19: 1439-1446.

Fein G, Meyerhoff DJ (2000). Ethanol in human brain by magnetic resonance spectroscopy: correlation with blood and breath levels, relaxation, and magnetization transfer. Alcoholism-Clin Exp Res 24: 1227-1235.

Fein G, Meyerhoff DJ, Weiner MW (1995). Magnetic resonance spectroscopy of the brain in alcohol abuse. Alcohol Health Res World 19: 3056-3314.
Foster-Gareau P, Heyn C, Alejski A, Rutt BK (2003). Imaging single mammalian cells with a $1.5 \mathrm{~T}$ clinical MRI scanner. Magn Reson Med 49: 968-971.

Grant SA, Millar K, Kenny GN (2000). Blood alcohol concentration and psychomotor effects. Br J Anaesthesia 85: 401-406.

Haase A, Frahm J, Hanicke W, Matthaei D (1985). 1H NMR chemical shift selective (CHESS) imaging. Phys Med Biol 30: 341-344.

Hahn RG, Norberg A, Jones AW (1997). 'Overshoot' of ethanol in the blood after drinking on an empty stomach. Alcohol Alcohol 32: 501-505.

Hanstock CC, Rothman DL, Shulman RG, Novotny EJ, Petroff OAC, Prichard JW (1990). Measurement of ethanol in the human brain using NMR spectroscopy. J Stud Alcohol 51: 104-107.

Hetherington H, Telang F, Pan J, Sammi M, Schuhlein D, Molina P et al (1999). Spectroscopic imaging of the uptake kinetics of human brain ethanol. Magn Reson Med 42: 1019-1026.

Heyn C, Bowen CV, Rutt BK, Foster PJ (2005). Detection threshold of single SPIO-labeled cells with FIESTA. Magn Reson Med 53: 312-320.

Hurd R, Sailasuta N, Srinivasan R, Vigneron DB, Pelletier D, Nelson SJ (2004). Measurement of brain glutamate using TE-averaged PRESS at 3T. Magn Reson Med 51: 435-440.

Hurd RE (2000). Magnetic resonance spectroscopic imaging having reduced parasitic side band signals. United States Patent no. 6069478 , May 30, 2000.

Hurd RE, Gurr D, Sailasuta N (1998). Proton spectroscopy without water suppression: the oversampled J-resolved experiment. Magn Reson Med 40: 343-347.

Kalant H (1971). Absorption, diffusion, distribution and elimination of ethanol: effects on biological membranes. In: Kissin B, Begleiter H (eds). The Biology of Alcoholism. Plenum Press: New York. pp 1-62.

Kalant H (2000). Effects of food and body composition on blood alcohol curves. Alcoholism-Clin Exp Res 24: 413-414.

Kaufman MJ, Chiu TM, Mendelson JH, Woods BT, Teoh SK, ErosSarnyai M et al (1996). Brain alcohol detectability increase with repeated administration in humans: a proton spectroscopy study. Magn Reson Med 35: 435-440.

Koob GF (2000). Neurobiology of addiction. Toward the development of new therapies. Ann NY Acad Sci 909: 170-185.

Krystal JH, Petrakis IL, Mason G, Trevisan L, D’Souza DC (2003). N-methyl-D-aspartate glutamate receptors and alcoholism: reward, dependence, treatment, and vulnerability. Pharmacol Therapeu 99: 79-94.

Lee H, Holburn GH, Price RR (2003). Proton MR spectroscopic studies of chronic alcohol exposure on the rat brain. J Magn Reson Imaging 18: 147-151.

Li TK, Beard JD, Orr WE, Kwo PY, Ramchandani VA, Thomasson HR (2000). Variation in ethanol pharmacokinetics and perceived gender and ethnic differences in alcohol elimination. AlcoholismClin Exp Res 24: 415-416.

Li TK, Lumeng L, McBride WJ, Waller MB (1979). Progress toward a voluntary oral consumption model of alcoholism. Drug Alcohol Depend 4: 45-60.

Lovinger DM (2002). NMDA receptors lose their inhibitions. Nat Neurosci 5: 614-616.

Mayer D, Spielman DM (2005). Detection of glutamate in the human brain at $3 \mathrm{~T}$ using optimized constant time point resolved spectroscopy. Magn Reson Med 54: 439-442.

Melendez RI, Hicks MP, Cagle SS, Kalivas PW (2005). Ethanol exposure decreases glutamate uptake in the nucleus accumbens. Alcoholism-Clin Exp Res 29: 326-333.

Moghaddam B, Bolinao ML (1994a). Biphasic effect of ethanol on extracellular accumulation of glutamate in the hippocampus and the nucleus accumbens. Neurosci Lett 178: 99-102.

Moghaddam B, Bolinao ML (1994b). Glutamatergic antagonists attenuate ability of dopamine uptake blockers to increase 
extracellular levels of dopamine: implications for tonic influence of glutamate on dopamine release. Synapse 18: 337-342.

Nebendahl K (2000). Routes of administration. In: Krinkle GJ (ed). The Handbook of Experimental Animals: The Laboratory Rat. Academic Press: San Diego. pp 463-483.

Neumark YD, Friedlander Y, Durst R, Leitersdorf E, Jaffe D, Ramchandani VA et al (2004). Alcohol dehydrogenase polymorphisms influence alcohol-elimination rates in a male Jewish population. Alcoholism-Clin Exp Res 28: 10-14.

O'Connor S, Morzorati S, Christian J, Li TK (1998). Clamping breath alcohol concentration reduces experimental variance: application to the study of acute tolerance to alcohol and alcohol elimination rate. Alcoholism-Clin Exp Res 22: 202-210.

Parks MH, Dawant BM, Riddle WR, Hartmann SL, Dietrich MS, Nickel MK et al (2002). Longitudinal brain metabolic characterization of chronic alcoholics with proton magnetic resonance spectroscopy. Alcoholism-Clin Exp Res 26: 1368-1380.

Petroff OAC, Novotny EJ, Ogino T, Avison M, Prichard JW (1990). In vivo measurements of ethanol concentration in rabbit brain by $1 \mathrm{H}$ magnetic resonance spectroscopy. J Neurochem 54: $1188-1195$.

Pfefferbaum A, Adalsteinsson E, Sullivan EV (2004). In vivo structural imaging of the rat brain with a $3 \mathrm{~T}$ clinical human scanner. J Magn Reson Imaging 20: 779-785.

Ramchandani VA, Bolane J, Li TK, O’Connor S (1999). A physiologically-based pharmacokinetic (PBPK) model for alcohol facilitates rapid BrAC clamping. Alcoholism-Clin Exp Res 23: 617-623.

Roberto M, Schweitzer P, Madamba SG, Stouffer DG, Parsons LH, Siggins GR (2004). Acute and chronic ethanol alter glutamatergic transmission in rat central amygdala: an in vitro and in vivo analysis. J Neurosci 24: 1594-1603.

Rooney WD, Lee JH, Li X, Wang GJ, Franceschi D, Springer Jr CS et al (2000). 4.0T water proton $\mathrm{T} 1$ relaxation times in normal human brain and during acute ethanol intoxication. AlcoholismClin Exp Res 24: 830-836.
Rossetti ZL, Carboni S (1995). Ethanol withdrawal is associated with increased extracellular glutamate in the rat striatum. Eur $J$ Pharmacol 283: 177-183.

Sammi MK, Pan JW, Telang FW, Schuhlein D, Molina PE, Volkow ND et al (2000). Measurements of human brain ethanol T(2) by spectroscopic imaging at 4T. Magn Reson Med 44: 35-40.

Schweinsburg BC, Taylor MJ, Alhassoon OM, Videen JS, Brown GG, Patterson TL et al (2001). Chemical pathology in brain white matter of recently detoxified alcoholics: a $1 \mathrm{H}$ magnetic resonance spectroscopy investigation of alcohol-associated frontal lobe injury. Alcoholism-Clin Exp Res 25: 924-934.

Seitz D, Widmann U, Seeger U, Nagele T, Klose U, Mann K et al (1999). Localized proton magnetic resonance spectroscopy of the cerebellum in detoxifying alcoholics. Alcoholism-Clin Exp Res 23: $158-163$.

Selim M, Bradberry CW (1996). Effect of ethanol on extracellular 5HT and glutamate in the nucleus accumbens and prefrontal cortex: comparison between the Lewis and Fischer 344 rat strains. Brain Res 716: 157-164.

Spielman DM, Glover GH, Macovski A, Pfefferbaum A (1993). Magnetic resonance spectroscopic imaging of ethanol in the human brain: A feasibility study. Alcoholism-Clin Exp Res 17: 1072-1077.

Srinivasan R, Sailasuta N, Hurd R, Nelson S, Pelletier D (2005). Evidence of elevated glutamate in multiple sclerosis using magnetic resonance spectroscopy at 3T. Brain 128: 1016-1025.

Tabakoff B, Hoffman PL (1996). Alcohol addiction: an enigma among us. Neuron 16: 909-912.

Webb P, Sailasuta N, Kohler S, Raidy T, Moats R, Hurd R (1994). Automated single-voxel proton MRS: technical development and multisite verification. Magn Reson Med 31: 365-373.

Widmark EMP (1981, originally published 1932). Principles and applications of medicolegal alcohol determination. Biomedical Publications: Davis, CA.

Yan QS, Reith ME, Yan SG, Jobe PC (1998). Effect of systemic ethanol on basal and stimulated glutamate releases in the nucleus accumbens of freely moving Sprague-Dawley rats: a microdialysis study. Neurosci Lett 258: 29-32. 